\title{
Jürgen Reulecke
}

\section{Utopische Erwartungen an die Jugendbewegung 1900-1933}

Wir haben jetzt noch zehn Jahre vor uns, um folgende Zukunftsvision Realität werden zu lassen, die im Herbst $1913 \mathrm{der}$ aus München stammende Wiener Philosophie- und Psychologieprofessor Friedrich Jodl als damals 64jähriger der Freideutschen Jugend auf dem Hohen Meißner in einem Geleitwort als ein „fernes Utopien“ für das Jahr 2013 ausmalte': Der enge Horizont des Christentums und das starre Festhalten an der klassischen Bildung seien dann überwunden; die geistigen Schätze der gesamten "Weltkultur“" d.h. die „ganze Fülle des innersten Lebens und geistigen Schauens, die sich in der Menschheit in Jahrtausenden erzeugt ${ }^{\text {“ }}$ habe, werde dann durch den Aufbruch der Jugend seit Beginn des 20. Jahrhun-

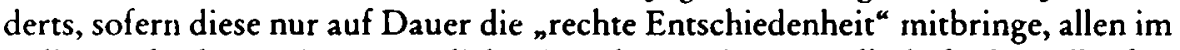
Volke in gleicher Weise zugänglich sein - dies in einer Gesellschaft, die völlig frei sei von Klassenegoismus und dem Machtstreben „einiger weniger bevorzugter Geister und Herrenmenschen “, frei auch von der "Rohheit des heutigen politischen und sozialen Kampfes “. Eine weltoffene neue Jugend werde dazu beigetragen haben, daß im Jahre 2013 „ein einiges starkes Deutschland“ besteht, „das der Mittelpunkt der Kulturwelt ist, nicht weil es die andern Völker beherrschte und ihnen das Joch seiner Sitten und Gesetze aufzwängte, sondern weil es den Geist menschheitlicher Kultur am vielseitigsten, reinsten und klarsten in sich ausgeprägt und anderen Völkern zum leuchtenden Vorbild hingestellt" habe.

Ähnlich wie Jodl, ein engagierter Kämpfer gegen Ultramontanismus und jede sonstige Form geistiger Gängelei aus den Kreisen des $W_{1}$ ener Liberalismus und Positivismus ${ }^{2}$, hatten sich im Frühherbst 1913 rund dreißig weitere geistige Köpfe der Zeit einerseits von dem Verleger Eugen Diederichs (geb. 1867) und andererseits von dem von der Deutschen Akademischen Freischar beauftragten Münchener Studenten Arthur Kracke (geb. 1890, also damals 23jährig) einladen lassen, Geleitworte zu einer Festschrift zum bevorstehenden ersten Freideutschen Ju-

1 Die folgenden Zitate sind dem Faksimileabdruck der Festschrift „Freideutsche Jugend. Zur Jahrhundertfeier auf dem Hohen Meißner 1913“ entnommen, in: Winfried Mogge, Jürgen Reulecke (Hrsg.), Hoher Meißner 1913 (Köln 1988) 165-169; im folgenden zitiert: Mogge, Reulecke, Festschrift. Der Titel des Beitrags von Jodl lautet: „1813-2013. Ein Programm“. 2 S. NDB 10 (Berlin 1974) $450 \mathrm{f}$. 
gendtag auf dem Hohen Meißner bei Kassel Mitte Oktober 1913 zu schreiben, darunter die Pädagogen Ludwig Gurlitt, Gustav Wyneken und Georg Kerschensteiner, die Philosophen Ludwig Klages und Paul Natorp, die Historiker Hans Delbrück und Robert von Pöhlmann, der Kultursoziologe Alfred Weber, die Schriftsteller Herbert Eulenberg und Ludwig Thoma, der Maler Fidus, der Mediziner Max von Gruber. Einige weitere angefragte Personen wie Gertrud Bäumer, Gerhart Hauptmann, Walter Goetz, Karl Lamprecht, Friedrich Naumann und Auguste Forel hatten zwar keinen Text geschickt, aber dem Vorhaben alles Gute gewünscht und freundschaftliche Grüße übermittelt. Ziel der Planer der Festschrift war es gewesen, „die bedeutendsten Führer des neuzeitlichen deutschen Geisteslebens zu Worte kommen (zu lassen), gewissermaßen in Ansprachen, mit Wünschen und Hoffnungen an die deutsche Jugend “ 3 .

Appelle dieser älteren Jugendbewegungfreunde, die sie im Zusammenhang mit dem Meißner-Treffen von 1913 formuliert haben, sollen im ersten Teil des vorliegenden Beitrags im Mittelpunkt stehen, ehe dann zunächst die frühen 20er Jahre und schließlich die Jahre um 1930 behandelt werden. Allerdings können hier zur Geschichte der Jugendbewegung und speziell jenes Treffens auf dem Hohen Meißner keine näheren Ausführungen gemacht werden; das würde den Rahmen sprengen ${ }^{4}$. Statt dessen soll es im folgenden um die generationsspezifischen Blickweisen gehen, die unter den Gesichtspunkten Zukunftshoffnung und -gefährdung von Beobachtern und Freunden jener Wandervögel und Freideutschen mit deren jugendbewegtem Aufbruch seit der Jahrhundertwende 5 und - später dann - mit der Bündischen Jugend der Weimarer Republik in Verbindung gebracht worden sind.

Zurück zunächst zum Umfeld des Meißner-Treffens vom Oktober 1913, das mit dem berühmten Gelöbnis endete: „Die Freideutsche Jugend will aus eigener Bestimmung, vor eigener Verantwortung, mit innerer Wahrhaftigkeit ihr Leben gestalten. Für diese innere Freiheit tritt sie unter allen Umständen geschlossen ein. "6 Durchgängig waren die von Diederichs und Kracke um Grußworte Gebe-

${ }^{3}$ Monatsbericht der Deutschen Akademischen Freischar (3/1913) 6; Zitat in: Mogge, Reulecke, Festschrift 72.

4 Vgl. die Einleitungsaufsätze zur Festschrift von Winfried Mogge und Jürgen Reulecke, 11-32, 33-62.

5 Des hundertsten Geburstags des Wandervogels wurde übrigens Anfang November 2001 mit einer Konferenz in Berlin-Steglitz gedacht: Im zweiten Hinterzimmer des dortigen Ratskellers ist am 4. November 1901 der "Wandervogel. Ausschuß für Schülerfahrten“" von etwa zwölf Personen gegründet worden, darunter neben ehemaligen Schülern des Steglitzer Gymnasiums, allen voran dem Abiturienten Karl Fischer (geb. 1881) und einem Mechanikerlehrling, fünf väterliche ältere Freunde, alle geboren in den 1850 er Jahren, von denen allein vier als ihren Beruf Schriftsteller angaben. Darunter war der wohl bekannteste der Schriftsteller und Propagandist der Heimatschutzbewegung Heinrich Sohnrey (geb. 1859); der Lehrer am Steglitzer Gymnasium Ludwig Gurlitt (s.u.) trat erst in den darauffolgenden Wochen hinzu. Siehe dazu Siegfried Copalle, Annalen des Ur-Wandervogels, in: ders., Heinrich Abrens, Chronik der Deutschen Jugendbewegung 1 (Bad Godesberg 1954) 13f.; vgl. auch Gerbard Ille, Günter Köhler (Hrsg.), Der Wandervogel. Es begann in Steglitz ... (Berlin 1987) $68 \mathrm{f}$.

6 Mogge, Reulecke, Festschrift bes. $50 \mathrm{ff}$. 
tenen keine Vertreter des wilhelminisch-nationalistischen oder militaristischen Establishments, sondern Personen, die als eher liberal-bürgerliche, z.T. sogar wie der Göttinger Privatdozent Leonard Nelson (geb. 1882) - sozialistischen Ideen zuneigende Zeitkritiker bekannt waren. Entsprechend fielen die meisten ihrer Beiträge aus, die durchweg auf eine dem Geist des deutschen Idealismus entspringende kulturelle Erneuerung der deutschen Nation durch die ,junge Generation“, vor allem durch die studentische Jugend, hinausliefen. Insofern schwangen immer auch Vorstellungen mit, die von utopischen Erwartungen im weitesten Wortsinn mitbestimmt waren, auch wenn manche der Autoren von aktuellen $\mathrm{Be}$ drohungen und befürchteten Fehlentwicklungen ausgingen. Die damalige Presse hat übrigens über die Äußerungen in der Festschrift wie auch über das Treffen auf dem Hohen Meißner bemerkenswert breit berichtet, wobei vor allem der folgende gereimte Festgruß des Düsseldorfer Schriftstellers und Dramaturgen Herbert Eulenberg (geb. 1876) die Gemüter bewegte und als - wie es hieß - „gehässiger Angriff" auf die Ehre der traditionsreichen studentischen Korporationen und schlagenden Verbindungen Anstoß erregte?:

„Ich grüße die Jugend, die nicht mehr säuft,

Die Deutschland durchdenkt und Deutschland durchläuft,

Die frei heranwächst, nicht schwarz und nicht schief.

Weg mit den Schlägern, seid wirklich, aktiv',

Das Mittelalter schlagt endlich tot!

Fin neuer Glauben tut allen not.

Bringt Humpen und Säbel zur Rumpelkammer,

Verjagt den Suff samt dem Katzenjammer

Und alles, was Euch verfault und verplundert!

Auf, werdet Menschen von unserm Jahrhundert!"

Es liegt selbstverständlich nahe, dic in Verbindung mit dem Meißner-Treffen an die jugendbewegten jungen Menschen gerichteten Appelle ihrer älteren Freunde und Gönner mit dem sich seit Ende des 19. Jahrhunderts sprunghaft ausbreitenden Jugendkult und Jugendmythos einerseits und mit den apokalyptischen Visionen angesichts der vorwiegend im Bildungsbürgertum verbreiteten Fin-de-siècleStimmung andererseits in Verbindung zu bringen. Tatsächlich finden sich, wenn man vom zeittypischen Pathos absieht, auch heute noch beeindruckende Zeitanalysen und Zukunftswarnungen in den eingesandten Texten. So prangerte z.B. Ludwig Klages (geb. 1872) in seinem Beitrag ${ }_{n}$ Mensch und Erde ${ }^{\alpha 8}$ vehement die durch die moderne Technik und den modernen Kapitalismus hervorgerufene, rasant voranschreitende Zerstörung der Natur an und setzte seine Hoffnung auf ein „kommendes Geschlecht“, welches „das Wissen von der weltschaffenden We-

7 Ebd. 145.

8 Ebd. 171-189; zu Klages vgl. Hinrich Jantzen, Namen und Werke. Biographien und Beiträge zur Soziologie der Jugendbewegung, Bd. 1 (Frankfurt a.M. 1972ff.) 149-154; im folgenden zitiert: Jantzen, Namen und Werke. Auch: Thomas Rohkrämer, Eine andere Moderne? Zivilisationskritik, Natur und Technik in Deutschland 1880-1933 (Paderborn u.a. 1999) 162-211; außerdem Diethart Kerbs, Jürgen Reulecke (Hrsg.), Handbuch der deutschen Reformbewegungen 1880-1933 (Wuppertal 1998) bes. $12 \mathrm{f}$. 
bekraft allverbindender Liebe " wiedergewinnen werde. Nur wenn - so Klages diese Liebe in der Menschheit "wiederwüchse, möchten vielleicht die Wunden vernarben, die ihr muttermörderisch der Geist geschlagen "9. Die in Deutschland besonders offensichtliche Nähe von Apokalypse und Utopie bestimmte also die Argumentation von Klages in ausgeprägter Weise ${ }^{10}$.

Andere Bedrohungen malte der Historiker Hans Delbrück (geb. 1848) ${ }^{11}$, mit Friedrich Jodl der älteste der Autoren, an die Wand. Geradezu prophetisch beschwor er die Gefahr, daß „die Welt einmal aufgeteilt (werde) zwischen englischem und russischem Wesen", und beklagte übrigens in diesem Zusammenhang schon damals das „ungeheure Übergewicht der englischen Sprache ${ }^{4}$ in der Welt ${ }^{12}$. Den Deutschen und besonders der deutschen Jugend wies er die Zukunftsaufgabe zu, sich als "Glied einer umfassenden Völker- und Kulturgemeinschaft" ${ }^{\text {" zu verste- }}$ hen und aus diesem Bewußtsein heraus die Vielheit der Kulturvölker gegen ,jene beiden Kolosse" zu verteidigen. Gleichzeitig warnte er die Jugend nachdrücklich vor zwei Zeitströmungen, denen er "ödesten Schematismus " vorwarf: Nationalismus und Sozialismus. Die Utopie eines sozialistischen Zukunftsstaates sei inhuman, weil dieser das Individuum ersticken und nuns damit in eine neue Barbarei stürzen würde". Jede Übersteigerung der nationalen Idee verenge dagegen den Gesichtskreis, trübe den Blick und sei der Feind jeder tieferen Bildung.

Der sechs Jahre jüngere Paul Natorp (geb. 1854) ${ }^{13}$, seit 1894 Sozialdemokrat und ein Gegner jeder Art von Rassenideologie, nach dem Kriege engagierter Pazifist, holte mit seinem Beitrag ${ }^{14}$ wohl am weitesten aus: $\mathrm{Er}$ wünschte sich durch eine Verallgemeinerung des jugendbewegten Wollens und Fühlens auf die Gesamtgesellschaft, vor allem des "schlichten Selbstseinwollens" der Jugendbewegten, nichts weniger als eine umfassende "Erneuerung des ganzen Lebens", eines nneuen Leben(s) der Deutschen, eine(s) Leben(s) der Natürlichkeit, Wahrhaftig-

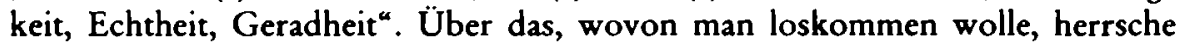
zwar, so Natorp, bei allen Reformern "hinreichende Klarheit", nicht jedoch über das, "was man erringen möchte“; und so mahnte er die Jugendbewegten, sich klar zu machen, daß sie der Lösung dieser gewaltigen Aufgabe nicht allein „mit Zupfgeige, neuer Kleidung und ähnlichen, an sich unverächtlichen Dingen“ beikommen könnten. Entsprechend breit fiel das Spektrum seiner Appelle und Visionen aus, die er der Jugend vom Hohen Meißner zum Bedenken anbot. Exemplarisch nur zwei Punkte: Wie Delbrück und Jodl beschwor auch Natorp das kulturelle ${ }_{n}$ Handinhandarbeiten der Kulturvölker des ganzen Erdenrunds“. Mit Kriegen

9 Mogge, Reulecke, Festschrift 188.

$10 \mathrm{Vgl}$. dazu Klaus Vondung, Die Apokalypse in Deutschland (München 1988); dort zu Klages 350f; im folgenden zitiert: Vondung, Apokalypse.

11 Zu Delbrück vgl. NDB 3 (Berlin 1957) 577 f.; siehe auch Rüdiger vom Bruch, Rainer $A$. Müller (Hrsg.), Historikerlexikon (München 1991) $67 \mathrm{f}$.

12 Mogge, Reulecke, Festschrift $140 \mathrm{f}$.

${ }_{13} \mathrm{Zu}$ Natorp s. NDB 18 (Berlin 1997) $752 \mathrm{f}$.

14 Titel seines Beitrags: „Aufgaben und Gefahren unserer Jugendbewegung “ in: Mogge, Reulecke, Festschrift 203-216. 
zwischen diesen Kulturvölkern - schon mit dem Gedanken an Krieg zu spielen, sei eine schwere Verantwortung! - sei deshalb kein nechter, innerer Ruhm ... zu holen“: „Denn ein heutiger Krieg, zwischen Völkern, die auf der Höhe heutiger, sich so nennender Kultur stehen, wäre nichts als ein technisches Exempel, in dem Menschen nur Ziffern oder Maschinenteile sind. Sein Leben wagen, das allein macht noch keinen Helden, zumal wenn so wenig von Freiwilligkeit dabei die Rede sein kann wie im Falle eines modernen Kriegs unter ,Kultur'-Völkern. “ Der zweite Punkt bezieht sich auf das, was Natorp "sittliche Reinheit" genannt hat: Scharf kritisierte er einerseits die moderne Großstadtzivilisation, andererseits die Formen einer - wie er zu beobachten glaubte - rasch um sich greifenden "Verwüstung des sexuellen Lebens". Mit einer „allgemeinen Gesundung des sexuellen Lebens" verband er geradezu eine "Rettung vom (drohenden) physischen und seelischen Untergang der deutschen Nation ". Hier könne nichts anderes mehr helfen, als "daß eine neue Jugend ersteht, fest und klar entschlossen, die Reinheit und Vornehmheit des Geschlechterverkehrs, die ehedem ein Hauptruhm und Stolz gerade unseres Volkes war, für sich wieder zu erringen und in unnachsichtiger Strenge zu bewahren". Die wenig später - 1916 - von Walter Flex in seiner in jugendbewegten Kreisen als eines der wichtigsten „Kultbücher" verehrten Schrift

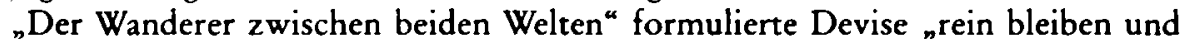
reif werden" ist also hier bereits wortgewaltig vorformuliert worden 15 !

Beim Lesen der Widmungsworte in der Meißner-Festschrift ist man versucht, möglichst viele der klingenden Zuschreibungen und Richtungsweisungen aus den Texten zu zitieren und sie - was auf der Hand liegt - mit der zeitgenössischen Debatte über den "neuen Menschen", die Aufgaben der Jugend im gerade angebrochenen "Jahrhundert des Kindes" und insbesondere die damals breit diskutierte Notwendigkeit umfassender Lebensreform in Verbindung zu bringen ${ }^{16}$. Eine Bemerkung in dem kurzen Gruß des Münchener Romanciers, Satirikers und Redakteurs beim "Simplizissimus" Ludwig Thoma (geb. 1867) zum Meißner-Fest lenkt jedoch den Blick auf eine bemerkenswerte Konstellation unter der Oberfläche der großen Worte und weitreichenden Visionen. Thoma wünschte sich von der neuen Jugend zunächst, daß sic sich von Eitelkeit, Selbstgefälligkeit und hohlen Phrasen freihalten, zur Pflichttreue erziehen und darum bemühen möge, ${ }_{n}$ Normen (zu) finden für allein echten Patriotismus", fügte dann aber noch den zunächst etwas verblüffenden Satz hinzu, er erhoffe sich von der neuen Generation vor allem, "daß sie in freier Menschlichkeit und in gerechter Würdigung des Nächsten den Großvätern ähnlicher werde als den Vätern “17. Thoma spielte mit dieser Bemer-

15 Siehe zu Flex Justus Ulbricht, Der Mythos vom Heldentod. Entstehung und Wirkungen von Walter Flex" „Der Wanderer zwischen beiden Welten“, in: Jahrbuch des Archivs der deutschen Jugendbewegung 16 (1986/87) 111-156.

16 Vgl. dazu Gottfried Küenzlen, Der Neue Mensch. Zur säkularen Religionsgeschichte der Moderne (München 21994); außerdem den Ausstellungskatalog „Der Neue Mensch. Obsessionen des 20. Jahrhunderts", hrsg. v. Nicola Lepp u. a. (Ostfildern 1999).

17 Mogge, Reulecke, Festschrift 247. 
kung auf eine in unserem Kontext, d.h. für die Frage nach den Zukunftsvisionen und deren Trägern, bedeutsame und seither immer wieder im 20. Jahrhundert aufbrechende Generationsproblematik an. Die Großväter waren für ihn jene „Gründergeneration", die das Deutsche Reich 1870/71 geschaffen und auf den Schlachtfeldern des deutsch-französischen Krieges gekämpft hatte. Mit den Vätern meinte er dagegen die um 1860 Geborenen, die als sog. "Wilhelminer", benannt nach Wilhelm II. (geb. 1859) ${ }^{18}$, in den letzten beiden Jahrzehnten vor dem Ersten Weltkrieg das öffentliche Leben und die Politik dominierten. Gegen deren aggressiv-autoritäres und unbeweglich-selbstsicheres Auftreten hatte sich bereits seit der Jahrhundertwende die wachsende Kritik einer jüngeren ( $Z$ wischen-)Generation gerichtet, die - geboren ab Ende der 1860er Jahre - mit den Fehlern dieser Wilhelminer abzurechnen begann und die Meißner-Jugend (geb. in den 1880er und 1890er Jahren) gegen deren Väter zu beeinflussen bzw. auf ihre Seite zu ziehen versuchte. Besonders massiv hat Arthur Moeller van den Bruck (geb. 1876) die Vorwürfe seiner Altersgruppe gegen die Wilhelminer formuliert und ihnen politische Inkompetenz und kulturelles Versagen vorgeworfen. „Die Schuld trifft nicht nur den Kaiser", schrieb er 1904, „sie trifft seine Generation mit, die sich ein Zeitalter wilhelminischer Laienhaftigkeit gefallen ließ“, und kam zu dem Schluß: „Ein Blutwechsel tut der Nation not, eine Empörung der Söhne gegen die Väter, die Ersetzung des Alters durch die Jugend. " 19

Es dürfte mehr als ein Zufall gewesen sein, daß zu den Autoren der MeißnerFestschrift kein einziger „echter “ Wilhelminer gehörte: Acht von ihnen zählten zu den Vertretern der vorhergehenden Altersgruppe (geb. um 1850), und die übrigen 21 Verfasser von Grußbotschaften waren deutlich jünger, nämlich fast alle nach 1867 geboren. Nur zwei Autoren mit eher unerheblichen Texten (der Verleger Karl Henckell und der Bildhauer Hermann Obrist, beide aus München) gehörten den Jahrgängen 1863 bzw. 1864 an. Entsprechend dieser klaren $Z$ weiteilung der Schreiber der "Freundesworte ${ }^{\text {in }}$ "Großväter" einerseits und etwa fünfzehn bis zwanzig Jahre ältere Freunde andererseits unterschieden sich auch die Inhalte und Stoßrichtungen der Appelle und der auf die Meißner-Jugend bezogenen Visionen. Ohne daß das jetzt hier breit belegt werden kann, läßt sich aus den Texten herauslesen, daß die an der Schwelle zum siebten Lebensjahrzehnt stehenden Älteren, wie die bereits zitierten Professoren Delbrück, Jodl und Natorp, aber auch die fünf anderen (Kerschensteiner, die Brüder Cornelius und Ludwig Gurlitt, der Althistoriker von Pöhlmann und der Mediziner von Gruber) intensive Beschwörungen an die Jugend formulierten, sich gegen den "Seelenfang" (Gurlitt) durch Staat, politische Parteien, Kirchen und Ideologien aller Art sowie gegen die vielfältigen massenzivilisatorischen Verführungen zur Wehr zu setzen, aber dabei nicht "die

18 Vgl. Martin Doerry, Übergangsmenschen. Die Mentalität der Wilhelminer und die Krise des Kaiserreichs (Weinheim, München 1986).

19 Ebd. 31f; Arthur Moeller van den Bruck, Die Deutschen, Bd. 1: Verirrte Deutsche (Minden 1904) 142; auch Barbara Stambolis, Der Mythos der jungen Generation (Diss. Bochum 1982) 28; im folgenden zitiert: Stambolis, Mythos. 
unabsehbar reiche Kulturarbeit der früheren Generationen" zu mißachten. Das Treffen auf dem Hohen Meißner schien ihnen ein Beweis zu sein, daß die jugendbewegten Teile des Nachwuchses auf dem richtigen Wege waren; in sie setzten sie ihre Hoffnungen, zumal - so Gurlitt - diese Jugend nicht mehr den ihr von oben vorgesetzten Führern folgen wolle, sondern "sich nach altgermanischer Sitte ihre Herzöge selbst küre“20. Das spätere Motto "Jugend soll durch Jugend geführt werden" klingt also bereits hier an. Selbstkritisch hatte Gurlitt schon vorher der jungen Generation die Aufgabe zugeschrieben, sie solle aus eigener Kraft aufbauen, „was wir haben verfallen lassen“21. Ähnlich wie Gurlitt betonten deshalb fast alle Autoren, daß die Jugend unbedingt ihren Weg allein finden müsse. Alfred Weber (geb. 1868) drückte diese Grundüberzeugung mit am klarsten aus, indem er schrieb: „Die größte Gefahr, die einer Jugendbewegung drohen kann, ist, nicht auf sich selbst zu stehen. Was sie will, muß sie fühlen, und was sie fühlt, das soll sie tun."22

Einige der jüngeren Autoren versuchten dennoch zumindest implizit, sich selbst als Wegweiser zu neuen Ufern zu empfehlen und entsprechende inhaltliche Perspektiven zu zeigen, so der Reformpädagoge Gustav Wyneken (geb. 1875) ${ }^{23}$ und die Schriftstellerin Gertrud Prellwitz (geb. 1869), übrigens die einzige Frau unter den Grußwortschreibern ${ }^{24}$. So bot sich z. B. Wyneken an, der Meißner-Jugend die Richtung zu einer " noch zu erarbeitenden neuen Jugendkultur" $\mathrm{zu}$ weisen, in der dann die verschiedenen lebensreformerischen Einzelbestrebungen ihren , allen gemeinsamen Gesichtspunkt" finden würden. Ins Zentrum der von ihm empfohlenen Zukunftsstrategie stellte er das Feld, das seiner Meinung nach am meisten für die Entstehung des von ihm scharf kritisierten Philistertums verantwortlich war: die Schule. Die neue Jugend - so Wyneken - solle sich die Schule als ihr "geistiges Heim" erobern; nur so werde sie der Welt wirkliche "Wiedergeburt und Verjüngung" schenken können und njene ersehnte innerliche Kultursynthese" zum Blühen bringen, "die frühere Zeiten Religion nannten “. Deshalb lautete sein Fazit: Dic Zeit des Plänkelns, des Spiels und der idyllischen Selbstgenügsamkeit sei nun vorüber; jetzt gelte es, „zum Angriff auf die Schule überzugehen; sie muss unser, sie muss der Jugend werden. Dieser Kampf wird mehr und mehr die nächsten Jahre und Jahrzehnte ausfüllen. " Und er fügte hintergründig hinzu: „Möge die Jugend ihren neuen Instinkt darin bewähren, daß sie ihre Führer zu finden weiß.“25 $\mathrm{Daß}$ er u. a. sich selbst damit meinte, belegt seine große Rede während des Meiß-

20 Mogge, Reulecke, Festschrift 161.

${ }^{21}$ Ludwig Gurlitt, Der Deutsche und sein Vaterland. Politisch-pädagogische Betrachtungen eines Modernen (Berlin 1902) 12.

22 Mogge, Reulecke, Festschrift 247.

${ }^{23} \mathrm{Zu}$ Wyneken siehe Jantzen, Namen und Werke 1, 321-328.

24 Zu Gertrud Prellwitz s. ders., Namen und Werke 4 (Frankfurt a.M. 1976) 229-234, außerdem Janos Frecot, Johann Friedrich Geist, Diethart Kerbs, Fidus 1868-1948. Zur ästhetischen Praxis bürgerlicher Fluchtbewegungen (Hamburg ${ }^{21997)}$ ) passim.

25 Mogge, Reulecke, Festschrift $250 \mathrm{f}$. 
ner-Festes und sollten dann seine vielen Schriften und Stellungnahmen in den nächsten Jahren zeigen ${ }^{26}$.

Die knappen Ausführungen von Gertrud Prellwitz in der Meißner-Festschrift mit dem Titel "Die Ehe und die neue Zeit" zielten auf eine weitere Handlungsperspektive, die der Meißner-Jugend nahegelegt wurde und Aufsehen erregen sollte ${ }^{27}$. Eher verklausuliert und vorsichtig als klar hatte sie den ,jungen, freudigen Frühlingskräften" des Meißner-Treffens nahegelegt, gerade auch im Bereich der Liebe und Erotik der eigenen "Linie der inneren Sicherheit " anstatt der äußeren "Linie der Konvention" zu vertrauen, wobei sie für die volle "Freiheit der lebensechten Innerlichkeit" im Bewußtsein hoher Selbstverantwortung und innerster Wahrhaftigkeit plädierte und jede Art von Zuchtlosigkeit scharf ablehnte. Ihre Vision lief auf eine Zukunft hinaus, in der die neuen Menschen ein „ernstes Verantwortungsgefühl gegenüber dem wichtigen, heiligen Lebensgute, das (die) Natur dem Menschen anvertraute, dem Liebesvorgang " entwickelt haben würden. Der Satz, der - aus dem Zusammenhang gerissen - in den folgenden Debatten den meisten Anstoß erregen sollte und zu der platten Unterstellung führte, die Prellwitz habe die Meißner-Jugend zur freien Liebe aufgefordert (zumal man auf dem Hohen Meißner auch für die Praktizierung der Nacktkultur eingetreten sei), lautete: „Wenn (sich) zum Beispiel die edlen jungen Kräfte der Quelle des Lebens, der Liebe nahen, da starrt ihnen etwas Todfremd-Feindliches entgegen: die heutige Form der Ehe. "Die folgende Fortsetzung des Gedankens unterschlugen dann allerdings die Kritiker, wenn sie empört die Prellwitz zitierten: „Die Ehe, wie das Gesetz sie uns bietet, wie die Sitte sie heiligt, legt das Schwergewicht auf den äußeren Schutz und Halt. Dadurch entsteht ein Zwang, ein beabsichtigter, und, unbeabsichtigt, eine sittliche Dumpfheit, Äußerlichkeit, Gedankenlosigkeit, Heuchelei, die jede heimliche Unredlichkeit zudeckt. ${ }^{28}$ In eher zurückhaltender Form hatte die Autorin hier also einen Gedanken vertreten und visionär in die Zukunft verlängert, der in den Kreisen der Frauenbewegung schon seit der Jahrhundertwende breit diskutiert worden war und auf eine "neue Ethik" in den Geschlechterbeziehungen hinauslief, in denen das freie Einverständnis beider Partner die Basis sein sollte29. Die fünfte Strophe des 1913 von dem Meißner-Fahrer Hermann Claudius (geb. 1878) ${ }^{30}$ gedichteten Textes des wohl mit am meisten gesungenen Jugendbewegungsliedes „Wann wir schreiten Seit' an Seit '“ griff gemäß

${ }^{26} \mathrm{Vgl}$. die Rede, die Wyneken auf dem Hohen Meißner gehalten hat (ebd. 293-301); sowie die als Broschüren von ihm seit 1913 in mehreren Auflagen verbreiteten Schriften "Die neue Jugend. Ihr Kampf um Freiheit und Wahrheit in Schule und Elternhaus, Religion und Erotik" (München 1913); und ders., Was ist "Jugendkultur"? (München 1913).

27 Vgl. Mogge, Reulecke, Festschrift 235-238; von ihr stammt auch ein weiterer Beitrag mit dem Titel "Was ist Vaterlandsliebe?" in: ebd. 232-235.

28 Ebd. $236 f$.

29 Siehe dazu Ute Frevert, Die Zukunft der Geschlechterordnung. Diagnosen und Erwartungen an der Jahrhundertwende, in: dies. (Hrsg.), Das Neue Jahrhundert. Europäische Zeitdiagnosen und Zukunftsentwürfe um 1900 (Göttingen 2000) 146-184, bes. $168 \mathrm{ff}$.

$30 \mathrm{Zu}$ Claudius s. Jantzen, Namen und Werke 1, 43-50. 
dem Refrainmotto „Mit uns zieht die neue Zeit“ genau diese Utopie auf und verkündete metaphorisch:

\author{
„Mann und Weib und Weib und Mann \\ Sind nicht Wasser mehr und Feuer. \\ Um die Leiber legt ein neuer \\ Frieden sich. Wir blicken freier, \\ Mann und Weib uns an."
}

Die in der Festschrift vehement vorgetragenen Visionen bzw. Versuche von Gustav Wyneken und Gertrud Prellwitz, die Meißner-Jugend für von ihnen empfohlene spezielle Ziele und Handlungsfelder zu begeistern, sind deshalb hier ausgewählt worden, weil sie ganz erhebliche Nachspiele haben sollten, denn sie schienen - so registrierten es vor allem Kreise in Bayern - massive Gefährdungen für die innere Stabilität, Religion und Sitte der Wilhelminischen Gesellschaft zu enthalten. Neben scharfen Reaktionen in einer Reihe von konservativen Zeitungen kam es Ende Januar 1914 im Bayerischen Landtag zu einer erregten Debatte, in welcher der Hauptredner, der Zentrumsabgeordnete und Bamberger Gymnasialprofessor Sebastian Schlittenbauer (geb. 1874), unterstützt durch viele weitere Abgeordnete aus fast allen Parteien und Kultusminister Eugen von Knilling (geb. 1865), das Urteil fällte: „Die Ziele dieser freideutschen Jugendkultur sind Kampf gegen das Elternhaus, gegen die Schule, gegen jede positive Religion und gegen den Patriotismus." ${ }_{11}$ Dem Minister rief Schlittenbauer abschließend unter dem Beifall des Hauses zu: Gegen diese modernen „destruierenden“ Bestrebungen könnten nkein schwächliches Paktieren ... und keine mattherzige Stellungnahme (helfen). Da heißt es: Nur immer feste druff!“ Und der liberale Abgeordnete Günther begründete die entschiedene Ablehnung der neuen Jugendkultur durch seine Partei mit den Worten: Liberalismus sei nicht Libertinismus; durch die neue Jugendkultur würden „nicht Männer von Charakter herangezogen, sondern aufgeblasene Frösche“, aber gerade die liberale Bewegung verlange ganze Männer ${ }^{32}$.

Es läge jetzt nahe, kann aber hier nicht unternommen werden, diese exemplarische Debatte am Vorabend des Ersten Weltkriegs detaillierter darzustellen. Ein erwähnenswerter Aspekt an ihr war jedenfalls, daß es neben Pauschalverurteilungen wie den oben zitierten vor allem scharfe Zurückweisungen der - meist aus dem Kontext gerissenen - Außerungen von Gustav Wyneken und Gertrud Prellwitz waren, die ahnen lassen, daß eine deutliche Kritik an den Verhältnissen in der Schule und in der bürgerlichen Familie und Ehe als massives Rütteln an den Grundfesten der damaligen Gesellschaft verstanden wurde. Schlittenbauer hat mit seinem Satz: „Das Volk hat nur eine Jugend zu verlieren; wenn diese verdorben ist, ist das ganze Volk verdorben“ die durch Äußerungen aus dem Umfeld des Meißner-Festes ausgelösten Ängste in der Vätergeneration recht eindeutig auf den Punkt gebracht. Wie in einem Brennspiegel zeigen diese bemerkenswerten Aus-

31 Vgl. die Broschüre „Die Freideutsche Jugend im Bayerischen Landtag (Hamburg 1914) hier: 3.

32 Ebd. 5. 
einandersetzungen in Bayern ebenso wie die vielfältigen entsprechenden Reaktionen in der damaligen Presse sowohl zum Meißner-Fest und seinem Umfeld als auch zu einer in diesem Kontext ebenfalls massiv ins Schußfeld geratenen "Zeitschrift der Jugend" mit dem Titel "Der Anfang “ neken zusammen mit dem Wiener Freud-Schüler Siegfried Bernfeld (geb. 1892) ${ }^{33}$, an welchen Frontlinien bzw. vor dem Hintergrund welcher generationsspezifischer Wahrnehmungen und Strategien die Frage nach den Zukunftsvisionen im Spätwilhelminismus verhandelt wurde. Es wäre reizvoll, darüber zu spekulieren, wie die Debatte weitergegangen wäre, wenn sie nicht im Sommer 1914 durch den Beginn des Ersten Weltkriegs in dieser kontroversen Form schlagartig überholt worden wäre.

Gegen die vielen Anwürfe und entstellenden Darstellungen in der Presse und besonders im Bayerischen Landtag mit ihren Folgen - der Wandervogel und die jugendbewegten sog. „Schülersprechsäle“ um die Zeitschrift „Der Anfang“ wurden in Bayern ebenso wie das Wandervogelliederbuch "Der Zupfgeigenhansl“ verboten - wehrte sich die Freideutsche Jugend, die in München besonders stark vertreten war, in einer bis dahin einmaligen Art: Sie veranstaltete in der Münchener Tonhalle am 9. Februar 1914 mit großem Erfolg eine sog. „Aufklärungsversammlung ", zu welcher der Heidelberger Kulturphilosoph und Soziologe Alfred Weber (geb. 1868) als Hauptredner gewonnen werden konnte und bei der Gustav Wyneken eine beeindruckende Verteidigungsrede hielt. Anwesend waren neben Siegfried Bernfeld und vielen Vertretern jugendbewegter Gruppierungen auch Ludwig Gurlitt, Ludwig Quidde und Arnold Zweig. Weber begrüßte in seiner Rede nachdrücklich die von der Freideutschen Jugend angestoßene Diskussion über Autorität und Freiheit und lobte die jungen Leute, weil sie "mit Dingen fertig geworden (seien), an die kein Mensch unserer Generation als spontane Leistungen der Jugend gedacht hat ${ }^{434}$. Er hielt ihre Aktionen für eine "geglückte Überrumpelung " der Wilhelminischen Gesellschaft und forderte die Freideutsche Jugend auf, sich durch die vielen öffentlichen Repressalien nicht zerbrechen zu lassen und auch weiterhin "nicht von außen nach innen, sondern von innen nach außen zu handeln", damit sie "jenes Geschlecht vielleicht schaffen könne", von dem Deutschland seit hundert Jahren geträumt habe.

Die an die Jugendbewegung herangetragenen hoffnungsfrohen Erwartungen älterer Freunde und die gleichzeitig mit ihrem Aufblühen verbundenen Befürchtungen und Bedrohungsszenarien waren nur die eine Seite der Medaille. Im Kontext dieses ersten Höhepunktes der Auseinandersetzung über Sinn und Richtung der Jugendbewegung um 1913 fühlten sich auch die inzwischen älter gewordenen Wandervögel und jungen Freideutschen selbst aufgerufen, ihre Bewegung zu in-

33 Siehe dazu KLaus Laermann, Der Skandal um den Anfang. Ein Versuch jugendlicher Gegenöffentlichkeit im Kaiserreich, in: Thomas Koebner, Rolf-Peter Janz, Frank Trommler (Hrsg.), "Mit uns zieht die neue Zeit ${ }^{*}$. Der Mythos Jugend (Frankfurt a.M. 1985) 360-381; im folgenden zitiert: Koebner u.a., Mythos Jugend. Vgl. auch Ulrich Hermann, Die Jugendkulturbewegung. Der Kampf um die höhere Schule, in: ebd. 224-245.

34 "Die Freideutsche Jugend im Bayrischen Landtag" (Hamburg 1914) 19. 
terpretieren. Seither entstand eine kaum mehr zu überschauende Flut von z.T. pathetischen Selbstdeutungen, aber auch gelegentlich durchaus selbstkritischen Äußerungen, die dann oft von den nachgeborenen Geschichtsschreibern und späteren Interpreten als Quellen zur Beschreibung angeblicher Realität oder als Ansatz zu massiver rückblickender Verurteilung benutzt werden konnten.

Eine zweite Welle zukunftsgerichteter Zuschreibungen an die Jugendbewegung entstand um 1920 und war Ausdruck jener Stimmungslage, die sich etwa auch in der Präambel des 1922 entworfenen Reichsjugendwohlfahrtsgesetzes folgendermaßen niedergeschlagen hat: Zur körperlichen, geistigen und sittlichen Gesundung und Erneuerung des niedergebrochenen deutschen Vaterlandes - so heißt es dort - müsse an erster Stelle bei der Jugend begonnen werden; sie solle befähigt werden, in Zukunft starke neue Kräfte zu entfalten ${ }^{35}$. Klaus Vondung hat u. a. am Beispiel des 1918 erschienenen ersten Buches von Ernst Bloch „Der Geist der Utopie" gezeigt, in welch ausgreifender Weise direkt nach Kriegsende apokalyptisches und utopisches Denken als Reaktion auf die tiefe gesellschaftliche Sinnkrise geradezu zu einer Art intellektueller Mode wurde ${ }^{36}$. Ernst Bloch (geb. 1885) hat übrigens später in seinem ab 1938 verfaßten Hauptwerk „Das Prinzip Hoffnung " die Jugendbewegung ausdrücklich in sein System der "Sozialutopien" einbezogen und sie als ein "Beweisstück für den fragmentarischen und realutopischen Charakter der , noch nicht' zur Heimat gewordenen Welt" bezeichnet ${ }^{37}$.

Besonders klingend äußerte sich nach Kriegsende 1918/19 der Züricher Ethikprofessor und Erziehungswissenschaftler Friedrich Wilhelm Foerster (geb. 1869) über die Jugendbewegung, indem er ihr bestätigte, sie sei „das Schneeglöckchen mitten im harten deutschen Winterschnee". Sie läute "den deutschen Frühling ein, sie (sei) ein wahrer Trost für jeden Deutschen, der schon daran verzweifeln wollte, daß sich die deutsche Seele jemals wieder aus der Verzauberung lösen werde, in die sie durch ihre Hinwendung zur Machtpolitik unwiederbringlich verstrickt schien $^{438}$. Und er wiederholte ein damals viel zitiertes Motto, das in diversen Abwandlungen noch mehrfach im 20. Jahrhundert ertönen sollte: „Los von den Sünden der Väter!“ Allerdings war Foerster bei aller Euphorie über den „freideutschen Mensch(en) “ als einen neuen Jugendtypus, der zweifellos „zu größten

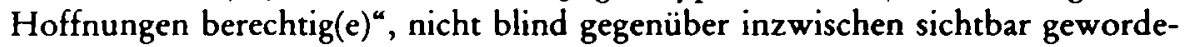
nen Fehlentwicklungen: Der jugendbewegte Mensch - so Foerster - sei nämlich in der Gefahr, nicht von sich selbst loszukommen und vor lauter Freude an sich selbst in einen inneren Stillstand, "nämlich in Selbsttäuschung und Phrasenmache-

35 Zum Reichsjugendwohlfahrtsgesetz siehe Christa Hasenclever, Jugendhilfe und Jugendgesetzgebung seit 1900 (Göttingen 1978) bes. 54 (zur Begründung des Gesetzentwurfs).

36 Vondung, Apokalypse bes. 225-257.

37 Siehe dazu Eckard Holler, Ästhetik des Widerstandes und politisches Engagement in der bündischen Jugend, in: Peter Ulrich Hein (Hrsg.), Künstliche Paradiese der Jugend (Münster 1984) 73-99, bes. 85 ff.; im folgenden zitiert: Holler, Ästhetik.

${ }^{38}$ Friedrich Wilhelm Foerster, Jugendseele, Jugendbewegung, Jugendziel (München, Leipzig 1923) 7. 
rei “ zu verfallen ${ }^{39}$. Diese Gefahr hat der selbst von der Wandervogelbewegung geprägte evangelische Pfarrer und spätere Bischof Wilhelm Stählin (geb. 1883) noch krasser charakterisiert, als er in einer weit verbreiteten Broschüre aus dem Jahre 1922 mit dem Titel "Fieber und Heil in der Jugendbewegung" sowohl das Motto "Los von den Sünden der Väter" als auch "diese ewige Umschmeichelung der Jugend, wie sie namentlich Gustav Wyneken leider geübt hat, " als "Verbrechen an der Jugend" kritisierte: Unter der "Firma des Wandervogels und der Jugendbewegung" seien auch ein ,jugendlicher Größenwahn und eine jeder Ehrfurcht bare Frechheit groß (geworden) ${ }^{40}$. Noch massiver äußerte er sich mit Blick auf aus rassistisch-völkischen Kreisen stammende Utopien, die der Jugendbewegung nahe legten, in "Rasse-Siedlungen" eine möglichst gesunde und reine Rasse "arischer Abstammung“ zu züchten. Diesen Gedanken hielt er für eine „barbarische Scheußlichkeit" und warnte die Jugendbewegten nachdrücklich davor, sich auf solche Experimente einzulassen ${ }^{41}$. Stattdessen forderte er sie auf, den Aufruf zu Tat und Formgebung als Aufruf zum politischen Handeln in dem Sinn zu verstehen, „daß sie im sozialen Ganzen wirken und kämpfen für das, was sie als Heil erkannt haben“. Was das im einzelnen sein sollte, erläuterte Stählin in auch heute noch beachtlicher Weise, doch produzierten viele andere Autoren gleichzeitig eine solche Fülle pathetischer und tiefsinnig gemeinter Ideen, daß der der Jugendbewegung eigentlich wohlwollend gegenüberstehende Philosoph Max Scheler (geb. 1874) schon 1923 bedauernd schrieb, die allzu ${ }_{n}$ gesteigerte Reflexion auf das Jungsein" und seinen Selbstwert und die oft bis zur Geistes- und Vernunftverachtung fortschreitende Überbetonung der, Lebens'- und ,Vital'werte gegenüber den Werten des Geistes" seien höchst bedenklich, und er fügte hinzu: "Ja, diese ewige Reflexion auf die Jugend - ist sie nicht selbst im Grunde unjugendlich?“ Aber auch Scheler setzte große Erwartungen darauf, daß das deutsche Volk im Sinne des "Stirb und Werde " mit Hilfe dieser Jugend seinen "kritischen Punkt " überwinden werde, und glaubre in der Jugendbewegung, nin ihrem Glauben, in ihrer Kraft, in ihrer Hoffnung ", den "geheimnisvolle(n) Vorgang der , Wiedergeburt' des geistigmoralischen Springquells“ der Nation zu entdecken ${ }^{42}$.

Die zweite Hälfte der 1920er Jahre erzeugte zwar auch weiterhin manche hochgestimmten Beschwörungen jugendbewegten Wollens, und in jugendbewegten Gruppen schwärmte man in selbstverfassten Liedern und Geschichten von ästhetisch ausgeschmückten Traumwelten, von "Eutopien" mit Namen wie "Thule“,

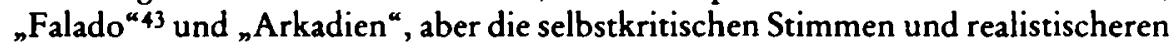
Beurteilungen nahmen zu. So beklagte z.B. 1927 der aus der Freideutschen Ju-

39 Ebd. 357.

40 Wilhelm Stäblin, Fieber und Heil in der Jugendbewegung (Hamburg 1922) $16 \mathrm{f}$.

41 Ebd. 78.

42 Max Scheler, Jugendbewegung, in: ders., Gesammelte Werke, Bd. 6 (Bern, München 21963) 396.

${ }^{43}$ Vgl. z. B. Jürgen Reulecke, Wo liegt Falado? Überlegungen zum Verhältnis von Jugendbewegung und Heimatbewegung, in: Edeltraud Klueting (Hrsg.), Antimodernismus und Reform (Darmstadt 1991) 1-19. 
gend stammende Jurist Normann Körber (geb. 1891) die „oft in blassen Ästhetizismus oder intellektuellen Formalismus ausartende Haltung der Freideutschen Jugend " und hielt ihr die gleichzeitige Vitalität der proletarischen Jugend entgegen. Enttäuscht stellte er fest, daß die Freideutschen „kein inhaltlich bestimmtes, lebendiges Bild vom Menschen aus sich herauszustellen vermocht (hätten) ${ }^{444}$. Der schon zitierte Wilhelm Stählin kritisierte gleichzeitig, daß die Jugendbewegung Jugendlichkeit als Wert an sich propagiert und damit den Weg der Jugend zur Reife "maßlos erschwert" habe: "Jugend obne den Blick auf die Reife, die den Heranwachsenden verantwortlich und mitgestaltend in diese Welt hineinstellt", sei letztlich bloß neine groteske Komödie " - so Stählin45. Die schärfste Kritik aber kam von außen und setzte ebenfalls an der Enttäuschung an, daß die utopischen Entwürfe und "der ungeheure Aufwand, der in der Jugendbewegung geleistet (werde), ... für die Umgestaltung der Gesellschaft in Deutschland fast vertan (gewesen sei) “: Unter den Titeln "Die tote Last" und „Alte Wandervögel" veröffentlichte 1926 Ignaz Wrobel alias Kurt Tucholsky (geb. 1890) in der "Weltbühne ${ }^{\alpha}$ zwei höchst bissige Artikel ${ }^{46}$, in denen er der Jugendbewegung vorwarf, sie sei bloß das gewesen, was deutsche Organisationswut, Reglementstorheit und Gruppenspiel immer gewesen (sei): Selbstzweck“ “. Der gewaltige „Seelenrummel“ sei „langsam verdunstender Dampf, der nie ein Rad getrieben ... eine Lokomobile, die das Holz sägt, mit dem sie gefeuert wird“, und er bedauerte, „wie viel anständige Gesinnung, wie viel wahrhaftiger Idealismus hier ... abgefangen, in eine Sackgasse geleitet (worden sei) “. Auf Tucholskys Fazit, „das Missverhältnis $z$ wischen den geradezu sektiererischen Aufregungen der Klüngel und ihrer tatsächlichen Wirksamkeit (sei) schlichtweg lächerlich“, antwortete ihm - ebenfalls in der "Weltbühne" - Max Barth, ein Vorkriegswandervogel, mit der desillusionierten Erklärung, um die großen Ziele der Jugendbewegung in der Gesellschaft erreichen zu können, hätte es einer nintakte(n) Generation Intakter mit geschlossenem Willen oder ein(es) große(n) Einzelne(n) ${ }^{a}$ bedurft; aus dem Krieg seien jedoch nur "Fragmente einer defekten Generation“ mit einem "seelischen Knacks" zurückgekommen, deren ehemalige "revolutionäre Bewegung" als Bewegung einer sunbürgerlichen Bürgerjugend" zudem bald durch die Alten ruiniert worden sei: vor allem durch Lebensreformer aller Bekenntnisse und durch Nationali$\operatorname{sten}^{47}$. In Form vieler versprengter „Individualitäten“ sei aber im Alltag durchaus

44 Normann Körber, Das Bild vom Menschen in der Jugendbewegung und unsere Zeit, wieder abgedruckt in: Grundschriften der deutschen Jugendbewegung, hrsg. v. Werner Kindt (Düsseldorf, Köln 1963) 472-487, hier: 481.

45 Wilhelm Stählin, Vom Schicksal und Sinn der deutschen Jugend (Wülfingerode-Sollstedt 1927) 68.

46 Ignaz Wrobel, Alte Wandervögel, in: Die Weltbühne 22 (1. Hj. 1926) 966-969; ders., Die tote Last, in: Die Weltbühne 22 (2. Hj. 1926) 855-857.

47 Max Barth, Die Letzten der Mohikaner, in: Die Weltbühne 22 (2. Hj. 1926) 966f.; s. auch die ebenfalls deutliche Selbstkritik des ehemaligen Jugendbewegten Max Peters, Partei der Jugend: Deutsche Linke, in: Die Weltbühne 21 (2. Hj. 1925) 736: Man habe erst nach 1923 zu entdecken begonnen, daß man "der Wirklichkeit gegenüber nichts vorzuweisen hatte“. Vgl. 
inzwischen ein neuer Typus Mensch wirksam, und dieser habe als Wertvollstes Kinder hervorgebracht, "die ein besserer Wurf und eine tüchtigere Sorte (seien), als wir seinerzeit gewesen sind“. Diese Kinder sollten dann später - so ist aus der Rückschau anzumerken - die "Hitlerjugendgeneration" genannt werden.

In den Älteren- und Führerkreisen der Jugendbewegung bahnte sich gegen Ende der 1920er Jahre aufgrund der genannten Erkenntnisse ein Trend an, der als Entwicklung ${ }^{2}$ von der Utopie zur Sozialreform" bezeichnet worden ist ${ }^{48}$. D.h. statt der Propagierung hochfliegender Perspektiven und utopischer Ziele engagierte man sich zunehmend - dies trifft besonders auf das Umfeld der "Deutschen Freischar" zu, eines 1927 erfolgten Zusammenschlusses mehrerer Wandervogelund Pfadfinderbünde - in sozialen Projekten und startete eine Reihe bemerkenswerter Experimente, so z.B. die Gründung freier Volksbildungsstätten, Landvolkshochschulen und eines Musikheimes, die Einrichtung von Wohnheimen und freiwilligen Arbeitslagern für arbeitslose Jugendliche u.ä. Allerdings führten diese Aktivitäten gleichzeitig dazu, daß sich ab 1928/29 die jüngere Jugendbewegungsgeneration der im letzten Jahrzehnt vor dem Ersten Weltkrieg Geborenen gegen eine solche "Politik“ ihrer Bundesführer auflehnte und rebellierte. Mit anderen Worten: Der offensichtliche Verlust utopischen Potentials in den Bünden provozierte neue Auf- und Ausbrüche, allen voran die des Eberhard Köbel, genannt tusk (geb. 1907), der Ende 1929 seine d.j.1.11 (deutsche jungenschaft vom 1.11.) gründete und damit die gesamte bündische Szene bis zum Verbot der Bünde 1933 für kurze Zeit in erhebliche Beunruhigung versetzte. Von ähnlich exemplarischer Bedeutung und ausstrahlender Wirksamkeit war gleichzeitig das ebenfalls 1929 gegründete "Graue Korps" Alfred (fred) Schmids (geb. 1899), der ausdrücklich die (Selbst)Erziehung in einem Jungenbund - verstanden als ${ }_{n}$ politisches Schutzgebiet" - als Voraussetzung dafür ansah, daß demnächst ein "Aufstand der jungen Generation“ ein neues Reich schaffen werde, in dem dann die besten Kräfte der bündischen Jugend mit den besten Kräften der Arbeiterjugendbewegung zusammenwirken würden ${ }^{49}$. Neben solchen Perspektiven waren es vor allem die mitreißenden neuen Stilformen und die ästhetisch überhöhte „Sozialutopie“ eines soldatisch-disziplinierten Jungenreichs unter der charismatischen Führung eines "großen Kameraden", die viele der damals 14 - bis 20 -jährigen begeisterten ${ }^{50}$ - dies

dazu auch Frank Trommler, Mission ohne Ziel. Über den Kult der Jugend im modernen Deutschland, in: Koebner u.a., Mythos Jugend 14-49, bes. $25 \mathrm{f}$.

48 Ortrud Wörner-Heil, Von der Utopie zur Sozialreform (Darmstadt, Marburg 1996).

49 Alfred Schmid, Aufstand der Jugend (Berlin 1930), hier zit. nach der Dokumentation der Jugendbewegung, Bd. 3, hrsg. von Werner Kindt (Düsseldorf 1974) 941-945; s. auch Stambolis, Mythos $287 \mathrm{ff}$. (zu Köbel und Schmid). Hans Mommsen hat in der Diskussion insbesondere auf einen von Alfred Schmid 1931 gedrehten Film über das "Graue Korps“ mit dem Titel "Der Ruf ${ }^{\alpha}$ hingewiesen, in dem eindrucksvoll in einer symbolischen Handlung die Utopie eines zukünftigen Zusammengehens des kämpferisch-soldatischen und des lyrischgeistigen Prinzips ästhetisch umgesetzt worden ist. Siehe zur Entstehung dieses Films Wilbelm Wald, Inseln der Unantastbarkeit (Heidenheim 1980).

$50 \mathrm{Vgl}$. Holler, Ästhetik $82 \mathrm{ff}$. 
mit diversen Nachwirkungen weit über den Zweiten Weltkrieg hinaus, aber auch z. B. für jugendliche Widerstandszirkel wie die "Weiße Rose“.

Der erwähnte Verlust an werbewirksamem Utopiegehalt in den inzwischen etablierten älteren Bünden der bürgerlichen Jugendbewegung hatte aber auch zur Folge, daß das plakative Utopieangebot der Nationalsozialisten, die zur Umwerbung der jungen Generation bedenkenlos jugendbewegte Stilmittel, Begriffe und Teilkonzepte kopierten und einsetzten, bei einer wachsenden Zahl junger Leute aus den Bünden Anklang fand, zumal Agitatoren aus der Frontkämpfergeneration wie Gregor Strasser (geb. 1892) mit seinem Slogan „Macht Platz, ihr Alten“ in der aufgeheizten Stimmungslage um 1930 Wege zu zeigen schienen, wie man das ungeliebte, „verknöcherte System“ von Weimar hinwegfegen konnte. Auch wenn radikale rechte Kreise vielerlei utopisch-völkische und rassistische Gedankengebäude produzierten und sie der Jugend anpriesen, so war letztlich das jetzt bewegende Element im wesentlichen ein immer hektischer werdender und auf Kampf drängender Aktionismus, der die - wie Peter Suhrkamp sie damals genannt hat ${ }^{51}$ "Söhne ohne Väter und Lehrer" mitriß: In der Zeitschrift "Die Tat" vom Juni 1930 hat Uttmann von Elterlein (geb. 1902) die historische Mission der pjungen Generation“ z.B. folgendermaßen beschworen: „Das Gesicht des XX. Jahrhunderts wird von denen geprägt, die dieses Verhältnis (oder Missverhältnis)“ - gemeint ist hier lt. Autor das aus früheren Jahrhunderten ererbe Verhältnis von Materialismus und Spiritualismus, von Rationalismus und Idealismus - „als Befehl zum Kämpfen empfinden. Also gehören Frontgeneration und alle folgenden Jahrgänge zusammen; nicht, weil sie wollen (das ist nebensächlich), sondern, weil die Geschichte so will“52. Und dieses Kämpfen sollte dann, so verkündeten die NSIdeologen, allen voran der Berliner Philosoph und Professor für politische Pädagogik Alfred Baeumler (geb. 1887), die Aufgabe disziplinierter Jungmännerbünde sein: Ihre innere Kraftquelle, aus der heraus der NS-Zukunftsstaat gespeist werden würde, sei ihr „heroischer Enthusiasmus“. Der Gegner, den diese Bünde allerdings vorher zu überwinden hatten, war laut Baeumler die bürgerliche Lebensform. Deshalb bestand seiner Meinung nach die Aufgabe der nationalsozialistischen Revolution darin, zunächst einmal das ,intimste deutsche Problem (zu) lösen“, nämlich dem Philisterium, d.h. der bürgerlich-liberalen Entartung des Männerbundes zu entgehen und ihn als „deutschen Männerbund ... aus der Tiefe unseres Seins und Wesens neu (zu erzeugen) ${ }^{a 3}$.

Indem also führende NS-Denker aus der ${ }_{n}$ Frontgeneration“ die Perspektive einer männerbündisch dominierten Zukunftsgesellschaft zum Programm erhoben und ihre Bewegung als eine „bündische “ deklarierten, köderten sie die vom Rückgang des utopischen Elans in den eigenen Bünden enttäuschten jüngeren Jugendbewegten aus der Generation der in den zwölf Jahren vor dem Ersten Weltkrieg

51 Peter Subrkamp, Söhne ohne Väter und Lehrer. Die Situation der bürgerlichen Jugend, in: Neue Rundschau 43 (1932) 681-692.

52 Uttmann von Elterlein, Absage an den Jahrgang 1902?, in: Die Tat 21 (Juni 1930) 206.

53 Alfred Baeumler, Männerbund und Wissenschaft (Berlin 1934) 34. 
Geborenen: Ihren ,liebgewordenen Idealvorstellungen von Jugendbewegung kam die scheinbare Übernahme der bündischen Lebensform als Struktur der Hitlerjugend, der Partei und Parteimiliz entgegen" - so lautete ein rückblickendes Urteil des Soziologen Theodor Geiger (geb. 1891) ${ }^{54}$. In eindrucksvoller Weise hat Geiger mit dem distanzierten Blick des Emigranten kurz nach dem Kriegsende 1950 - die spezifische Vereinnahmung der bündischen Idee und - umgekehrt - die mentalitätsgeschichtlichen "Vorleistungen" der Bünde in der Zeit der Weimarer Republik in Richtung Nationalsozialismus analysiert. Die Utopie einer Volksgemeinschaft ohne Klassengegensätze und Parteienzwist sei - so Geiger - gerade wegen ihrer Verknüpfung mit der "Sozialutopie“ des bündischen Gemeinschaftslebens für die jugendbewegten ,junge(n) Träumer und alte(n) Utopisten" so bestechend gewesen. Allerdings ging - wie bei manchen anderen Schlagworten, welche die Nationalsozialisten für sich okkupierten - mit der Vereinnahmung des Bündischen auch eine Pervertierung des bisherigen Inhalts einher, denn die elitäre bündische Lebensform war mit den autoritären NS-Massenorganisationen eigentlich nicht vereinbar. Daß sich viele Bündische der Frontgeneration und der Vorkriegsgeneration dennoch auf diesen Etikettenschwindel einließen, habe - so Geiger - mit der nationalsozialistischen Verwandlung des „einfühlsamen Expressionismus der Jugendbewegung ... in atemloses Pathos und barbarischen Heroismus" zusammengehangen.

Wenn Geiger in diesem Zusammenhang von "Expressionismus" spricht, spielt er auf jenen expressiven Ästhetizismus bzw. ,ästhetischen Fundamentalismus “ 55 an, der im Grunde auch das, was die Jugendbewegung an utopischem Denken produziert hat oder was ihr als solches unterstellt worden ist, weitgehend ausmachte. Denn abgesehen von jenen oben zitierten, anläßlich des Meißner-Festes von 1913 von den damaligen "Großvätern" formulierten Zukunftshoffnungen an den jugendbewegten Aufbruch der Freideutschen und der Wandervögel gerieten die meisten utopischen Zuschreibungen an die Jugendbewegung in der Folgezeit weitgehend zu einer ästhetisch ausgeschmückten Jugendmetaphysik ${ }^{56}$. Symbole, Stilisierungen, Ritualisierungen sowie die Beschwörungen eines farbig ausgemalten Arkadiens ${ }^{57}$ bestimmten die Vorstellungen über ein in der Zukunft herzustellendes humanes Zusammenspiel von Gesellschaft bzw. Kultur und Natur weit mehr als der Versuch, sich rational Rechenschaft über Sein, Sollen und Wollen ab-

54 Theodor Geiger, Die Gesellschaft zwischen Pathos und Nüchternheit (Kopenhagen 1950) 21.

55 Siehe dazu Stefan Breuer, Ästhetischer Fundamentalismus. Stefan George und der deutsche Antimodernismus (Darmstadt 1995).

56 Vgl. Peter Ulrich Hein, Jahrhundertwende, Kunstenthusiasmus und Jugendbewegung, in: ders. (Hrsg.), Künstliche Paradiese der Jugend. Zur Geschichte und Gegenwart ästhetischer Subkultur (Münster 1984) 24-29 (Unterkapitel „Ästhetizismus statt Utopie“).

57 Joachim Wolschke-Bulmabn, Auf der Suche nach Arkadien. Zu Landschaftsidealen und Formen der Naturaneignung in der Jugendbewegung (München 1990); s. auch Jost Hermand, Grüne Utopien in Deutschland. Zur Geschichte des ökologischen Bewußtseins (Frankfurt a.M. 1991); sowie ders., Der alte Traum vom neuen Reich. Völkische Utopien und Nationalsozialismus (Frankfurt a.M. 1988). 
zulegen, wie ihn etwa Wilhelm Stählin gefordert hatte. Bereits die Tatsache, daß Jugend nach dem Motto "Jugend ist eine Haltung, nicht eine Sache der Lebensjahre" als die entscheidende utopiefähige Kraft in der Gesellschaft vom Lebensalter abgekoppelt wurde, belegt, daß vor allem Ästhetizismus der Bewegungsmotor war, der die Beschwörungen und Deutungen der Jugendbewegung in der Zwischenkriegszeit letztlich antrieb, und nicht Utopie im engeren Wortsinn. Daß damit zugleich auch eine weitgehende inhaltliche Beliebigkeit verbunden war, hat Karl Jaspers in seiner bekannten Schrift "Die geistige Situation der Zeit" aus dem Jahre 1931 deutlich gemacht: „Hat der Mensch eigentlich kein Lebensalter mehr, so fängt er stets von vorn an und ist stets am Ende: er kann dies tun und auch das, und einmal dies, ein andermal jenes; alles scheint jederzeit möglich zu sein, nichts eigentlich wirklich. ${ }^{458}$

Dennoch gab es jugendbewegte Zirkel, die sich seit etwa 1929/30 darum bemühten, ein auf kritischer Befragung beruhendes, differenziertes Verhältnis zum Nationalsozialismus zu gewinnen. Ein Beispiel dafür ist die Münchener Hochschulgilde "Greif", in der um 1930 der damals 22-jährige Theodor Schieder (geb. 1908) "Gildenmeister" war. Hier wurde angesichts der immer stärker bewußt werdenden Herausforderung durch den Nationalsozialismus und angeregt durch die Argumente der "konservativen Revolution", wie sie vor allem im Tat-Kreis diskutiert wurden, intensiv über die Situation des Parteiensystems, die "Krise des Parlamentarismus" und die Zukunftsoptionen der ,jungen Generation " debattiert. Trotz einiger Sympathie zur nationalsozialistischen Bewegung und trotz deren ,innerer Verwandtschaft mit dem, was man unter bündischer Bewegung versteht ${ }^{\alpha}$, betonte Schieder in einem Grundsatzartikel im ${ }_{n}$ Rundbrief ${ }^{*}$ der Gilden die Unterschiede und vor allem die Notwendigkeit des Entwurfs von Zukunftsperspektiven über den von den Nationalsozialisten plakativ und demagogisch vorgetragenen "Generalnenner" hinaus: Dieser bestehe im Grunde nur aus einigen "großen Negationen"! Von den Nationalsozialisten würden bloß „Absolutierungen vorgenommen und die eigentlich wirksamen historischen Kräfte dahinter verschleiert. Und Lösungen, die nur Durchgangslösungen sind, vorläufige Mittel, die Formen des bestehenden Staates zu bekämpfen, (würden) als Endlösungen ausgegeben“. Das Fazit Schieders lautete deshalb: „Für uns beginnt dahinter erst das feste Neuland. "59 Die eigentlich zukunftsträchtige NS-Bewegung - so Schieder - sei noch viel zu sehr "mit einem in der Vergangenheit verhafteten und mit ihren Begriffen arbeitenden System belastet ${ }^{\text {" }}$; in ihr sei „die wilhelminische Aera noch nicht überwunden ". Hier klingt ein Gedanke an, den auch führende Sprecher des Tat-Kreises wie Hans Zehrer (geb. 1899) und Ernst Wilhelm Eschmann

58 Karl Jaspers, Die geistige Situation der Zeit (zuerst 1931 Berlin, hier zitiert nach dem Abdruck der im Sommer 1932 bearbeiteten 5. Aufl. Berlin, New York 1979) 44.

59 Theodor Schieder, Unsere Stellung zum Nationalsozialismus, in: Gemeinsamer Rundbrief der Gilden des Arbeitsabkommens, 1. Folge (Februar 1930) 16-22. Siehe dazu auch Jürgen Reulecke, Hat die Jugendbewegung den Nationalsozialismus vorbereitet? in: ders., „Ich möchte einer werden so wie die..." Männerbünde im 20. Jahrhundert (Frankfurt a.M. 2001) 151-176, bes. 153 ff.; im folgenden zitiert: Reulecke, Männerbünde. 
(geb. 1904) - beide aus der Jugendbewegung stammend - um 1930 vertraten, daß nämlich der Nationalsozialismus nur eine Übergangsstufe sei: Als "Vierte Front" werde die "junge Generation “ anschließend - voraussichtlich von den 1940er Jahren an, so Eschmann - die eigentliche Wende herbeiführen ${ }^{60}$.

Es ist bezeichnend, daß die sich in der Machtergreifungsphase 1932/33 an die studierende Jugend wendenden NS-Professoren wie der Pädagoge Ernst Krieck (geb. 1882) und der Philosoph Alfred Baeumler (geb. 1887) letztlich ästhetisch argumentierten, wenn sie die große Bedeutung betonten, die der Nationalsozialismus der Zeremonie und "dem Symbolischen in Wort und Tat ${ }^{4}$ beimesse ${ }^{61}$. "Haltung" war wichtiger als Inhalt, und die Absage an die Ratio gipfelte dann in solch lapidaren Sätzen wie dem folgenden von Joseph Goebbels (geb. 1897): „Es ist nicht so sehr von Belang, woran wir glauben; nur daß wir glauben!“62 - ein Motto, das sich in vielen Varianten z. B. auch in den zündenden NS-Marschliedern und in den von Autoren aus der "Jahrhundertgeneration " geschaffenen Liedern für die Hitlerjugend wiederfindet (z.B. in Liedtexten von Heinrich Anacker, Werner Altendorf, Herybert Menzel, Gerhard Schumann, Baldur von Schirach und vor allem Hans Baumann ${ }^{63}$ ). Die NS-Pädagogik schloß sich dieser Argumentation an: Die Erziehung in allen Männerbünden des NS-Staates von der Hitlerjugend bis zur SA, zur SS und zum Heer werde bestimmt sein - so der Erziehungswissenschaftler Karl Friedrich Sturm 1933 - von der „Vorherrschaft der leiblichen und Willenserziehung“, und er fügte kurz und unmißverständlich hinzu: „Geistige Schulung und insbesondere Bildung stehen zurück." 64

$\mathrm{Da} B$ die genannten Formen der Umwerbung der Jugendbewegten aus der seit etwa 1902 geborenen Kriegsgeneration nach der "Machtergreifung" durch die Wortführer der Frontgeneration überflüssig geworden waren und dann auch sofort ad acta gelegt wurden, ist vielfältig nachweisbar: $Z_{w a r}$ seien der italienische Faschismus wie der deutsche Nationalsozialismus , vom heißen Atem der Jugend erfüllt", verkündete z.B. Goebbels 1934, aber die Jugend gehöre dem Staat und müsse entsprechend diszipliniert werden ${ }^{65}$. Jugend besaß nun kein Eigenrecht mehr und gehörte für ihn zur "Rohstoffmasse Mensch", aus der die schöpferische Einzelpersönlichkeit in der Art eines Künstlers, der große Politiker, der "Führer" also, ein Volk formen und dieses dann $\mathrm{zu}$ "nationalpolitischer Bedeutsamkeit"

$60 \mathrm{Vgl}$. Hans Mommsen, Generationskonflikt und Jugendrevolte in der Weimarer Republik, in: ders., Von Weimar nach Auschwitz. Zur Geschichte Deutschlands in der Zwischenkriegszeit (Stuttgart 1999) 59-72, bes. $68 \mathrm{f}$.

61 Vondung, A pokalypse 213.

62 Zit. ebd. 224; aus Joseph Goebbels, Michael. Ein deutsches Schicksal in Tagebuchblättern (München 1929) 31.

${ }^{63}$ Siehe dazu Jürgen Reulecke, „Wir reiten die Sehnsucht tot“ oder: Melancholie als Droge, in: ders., Männerbünde bes. $116 \mathrm{f}$.

64 Karl Friedrich Sturm, Deutsche Erziehung im Werden (Osterwieck, Berlin 1933, hier zitiert nach der 4. Auflage 1938) $141 \mathrm{f}$.

65 Joseph Goebbels, Der Faschismus und seine praktischen Ergebnisse (Berlin 1934) bes. 27 und 29. 
emporheben werde 66 . Und in einer programmatischen Broschüre mit dem bezeichnenden Titel „Schluss mit ,junger Generation“!“ aus dem Jahre 1933 ist zu lesen, die junge Mannschaft sei zwar "kraft ihrer Lebendigkeit und ihrer Hingabebesessenheit“ zu zweierlei fähig, nämlich zur Zerstörung und zur Eroberung; Staatsgründung und Volksformung seien dagegen Aufgaben des Mannes! Es gehe deshalb jetzt um "die notwendige, endliche Rückführung aus atavistischer Besessenheit zu den ewigen Aufgaben der Menschen, die jeder an seiner Stelle still und bescheiden zu lösen (habe), die Verpflichtung zur Ordnung im allerengsten Umkreis des Persönlichen, den Jünglinge und Jüngling bleibende Männer allzu gern überspringen "67. Das Besondere an diesem Text ist, daß der Verfasser Karl Rauch (geb. 1897) aus der bündischen Pfadfinderschaft stammte, mit Saint-Exupéry (geb. 1900) befreundet war, sich in den 20er Jahren als „Gauherzog“ bei den Neupfadfindern in Mitteldeutschland engagiert hatte und insofern ein Beispiel dafür ist,

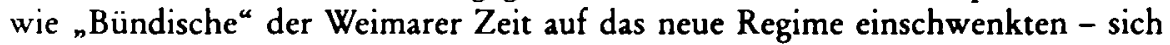
andienend bzw. in der Erwartung, daß ihre bündischen Ideale nun in die Tat umgesetzt werden könnten. Die gesamte Broschüre - insofern ist sie eine besonders exemplarische Quelle - ist von Hoffnung auf eine neue Zeit, aber auch von dem Bewußtsein eines Ausgeliefertseins bestimmt: Teig sei das Volk in des Führers Hand, schrieb auch Rauch metaphorisch, und er, der Führer, könne es (!) kneten. Was er daraus formen werde, liege an ihm. Was daraus folge, sei Sache des Glaubens und der Hoffnung, und er fügte dann - mehrdeutig - hinzu: „Es gibt nichts anderes. "68 Der Text endet mit der nüchternen Aufforderung an die Jugend und besonders die Jugendbewegten, sich als "Stand der jungen Mannschaft ${ }^{\text {“ }}$ in Volk und Staat einzuordnen, sich der Führung jener Männer anzuvertrauen, deren Werk das neue Staatswesen sei, und sich durch „Bereitung und Schulung a auf "Dienst und Bewährung “ als Mann in einem bisher nur erträumten „kommenden Reich" vorzubereiten ${ }^{69}$.

Kurz zusammengefaßt: Was die utopischen Erwartungen im frühen 20. Jahrhundert an die um 1900 mit dem Wandervogel und dann mit der Freideutschen Jugend ins Leben getretene bürgerliche Jugendbewegung angeht, spannt sich in den zwanzig Jahren von 1913 bis 1933 ein weiter Bogen von den enthusiastischen Hoffnungen, die junge Generation werde im 20. Jahrhundert frei und selbstbewußt von „innen“ heraus die Weichen für eine humanere Zukunftsgesellschaft stellen und den ,neuen Menschen“ schaffen, bis hin zur massiven Einhegung aller jugendlichen Bewegungskräfte in einem diktatorischen Erziehungsstaat. Dieser Staat, im wesentlichen gegründet von der „Frontgeneration“, instrumentalisierte in umfassender Weise die Jugend für seine Zwecke (Motto: „Auch Du gehörst dem Führer!“ $)$ und begann gleichzeitig seine utopischen Vorstellungen von einer rassereinen Volksgemeinschaft der Zukunft mit brutalen Methoden zu verwirk-

66 Ebd. 7.

67 Karl Rauch, Schluß mit njunger Generation“" (Leipzig 1933) Titelseite, sowie 51, 63.

68 Ebd. 47.

69 Ebd. $119 \mathrm{f}$. 
lichen. Er versicherte sich dabei gerade jener njungen Generation", die als "Jahrhundertgeneration" - von hohen Erwartungen begleitet - im letzten Jahrzehnt vor dem Ersten Weltkrieg geboren war und - als revolutionäre Erneuerungskraft umworben - ihre Jugend z.T. in jugendbewegt-bündischen Gruppen der Weimarer Republik erlebt hatte. Die Bedeutung dieser Altersgruppe für die Mentalitätsgeschichte der frühen Bundesrepublik, d.h. die sich daran anschließende Frage nach deren „mentalem Gepäck" und der Art und Weise, wie nicht zuletzt jugendbewegt geprägte Angehörige dieser Generation wie Theodor Schieder, Werner Conze, Günther Franz und Ernst Rudolf Huber nach 1945 eine „Verwandlungszone" (Gottfried Benn) durchlaufen haben, hat ja in jüngster Zeit insbesondere die Historikerzunft intensiv beschäftigt ... 